\title{
Magnetic gaps in organic tri-radicals: from a simple model
}

\author{
to accurate estimates
}

\author{
Vincenzo Barone ${ }^{a}$, Ivo Cacelli ${ }^{b}$, Alessandro Ferretti ${ }^{c^{*}}$, Giacomo \\ Prampolini $^{c}$
}

date: $24 / 05 / 2017$

a) Scuola Normale Superiore di Pisa, Piazza dei Cavalieri, I-56126 Pisa, Italy

b) Dipartimento di Chimica e Chimica Industriale, Università di Pisa, Via G. Moruzzi 13, I-56124, Pisa, Italy

c) Istituto di Chimica dei Composti OrganoMetallici (ICCOM-CNR), Area della Ricerca, via G. Moruzzi 1, I-56124 Pisa, Italy

\begin{abstract}
The calculation of the energy gap between the magnetic states of organic poly-radicals still represents a challenging playground for quantum chemistry, and high-level techniques are required to obtain accurate estimates. On these grounds, the aim of the present study is twofold. From the one side, it shows that, thanks to recent algorithmic and technical improvements, we are able to compute reliable quantum mechanical results for the systems of current fundamental and technological interest. From the other side, proper parameterization of a simple Hubbard Hamiltonian allows for a sound rationalization of magnetic gaps in terms of basic physical effects, unraveling the role played by electron delocalization, Coulomb repulsion and effective exchange in tuning the magnetic character of the ground state. As case studies, we have chosen three prototypical organic tri-radicals, namely 1,3,5-trimethylenbenzene, 1,3,5-tridehydrobenzene and 1,2,3tridehydrobenzene, which differ either for geometric or electronic structure. After discussing the differences among the three species and their consequences on the magnetic properties in terms of the simple model mentioned above, accurate and reliable values for the energy gap between the lowest quartet and doublet states are computed by means of the so-called difference dedicated configuration interaction (DDCI) technique, and the final results are discussed and compared to both available experimental and computational estimates.
\end{abstract}

* corresponding author e-mail: alessandro.ferretti@pi.iccom.cnr.it 


\section{Introduction}

The magnetic properties of matter are at the heart of several devices of routine use in our life, like, for instance, electric motors, magnetic tapes, diagnostic devices, computer memories and data storages. Pushed by the need of device miniaturization, there has been recently a strong impulse to the research in the field, following the idea of exploiting molecules or molecular aggregates, rather than bulk materials[1-9]. The discovery of the giant magneto-resistive effect by Peter Grunberg and Albert Fert is paving the route toward the construction of a new generation of hard disks and spintronic devices (see for instance References [[1019]], just to cite few recent examples), and this has, in turn, stimulated new experimental and theoretical investigations of magnetic molecular species.

As is well known, magnetism is the consequence of the presence of unpaired electrons, which are common in transition metals (the conventional route to magnetic materials), but quite unusual in stable organic systems (the so-called mono and poly-radicals). In particular, poly-radicals have attracted great attention,[2, 20-33] in view of technological applications that require spin-dependent transport and durable data storage[13, 34] or for application in batteries.[35] The ability to unravel and control the quantities at the origin of the spin-spin interactions in such systems is one of the key points for governing the magnetic properties of matter at the molecular scale. In this perspective, besides the considerable experimental effort that is often required to synthesize and stabilize poly-radical species [31, 36, 37], the contribution of theory and computation can be significant.

Much work has been devoted to the simplest members of the class, namely di-radicals, both from an experimental and a theoretical point of view. In fact, these compounds have been often employed as benchmarks to develop and test methods or models that might be possibly extended to more complex systems. Experimentally, these species, mostly based on nitroxide, nitronyl nitroxide and verdazyl units, can be managed without an excessive burden, whereas their relatively small dimensions allow for accurate and reliable computational investigations (besides the already cited work and references therein, some further references can be found in [38-48]).

In a di-radical, two unpaired electrons are localized in different parts of the molecule. The simplest model to describe a di-radical is the Heisenberg-Dirac-Van Vleck Hamiltonian, where the two electrons, interacting by a spin-spin coupling term $\mathrm{J}$, give rise to a singlet and a triplet state, $\mathrm{J}$ corresponding to their energy gap $(\mathrm{J}=$ $\left.\Delta \mathrm{E}_{\mathrm{ST}}=\mathrm{E}_{\mathrm{T}}-\mathrm{E}_{\mathrm{S}}\right)$. [1,29] A positive value of $\mathrm{J}$ is associated with an antiferromagnetic ground state, a negative one with a ferromagnetic, triplet, ground state. $\mathrm{J}$ is a quantity measurable by different techniques,[27] nonetheless, its computation is not trivial, since the singlet eigenstate has an intrinsic multi-reference character. The computational methods developed to deal with this situation have been extensively reviewed in Ref. [[29]] and belong to two main classes, rooted into the Density Functional Theory (DFT) and into the wavefunction (WF) description, respectively. Among DFT methods, the broken symmetry approach pioneered by Noodleman is still the most widely applied [49-52], although the quite recent Spin Flip-Time Dependent DFT[53-56] appears more reliable and theoretically sound. Within the WF approach, magnetic interaction in poly-radicals have been studied by several different multi-reference perturbative methods (such as CASPT2 
and NEVPT2 [57, 58], state specific MRPT [59] or by RAS-SF [60-63] and Coupled Cluster methods, such as the spin-flip EOM [48, 64]). In our opinion the Difference Dedicated Configuration Interaction (DDCI) method, originally proposed by Miralles et al in Ref. [[65, 66]], represents an excellent compromise between accuracy and computational feasibility, especially for large systems (see [29] and references therein), where an accurate choice of the active magnetic orbitals can improve the quality of the results [67].

In the past few years we have contributed to the field, proposing a computational route based on DDCI that takes advantage from orbital localization, virtual orbital reorganization and Möller-Plesset perturbation theory, which has been successfully applied to some nitroxide and nitronyl nitroxide diradicals [47, 68-71]. This computational procedure has been gathered in a comprehensive user-friendly software, named BALOO [47]. As a step forward, here we extend our approach to organic tri-radicals, focusing in particular on three prototypical species, 1,3,5-trimethylen benzene (TMB), 1,3,5-tridehydrobenzene (MM-TDB) and 1,2,3-tridehydrobenzene (OO-TDB), displayed in Figure 1. The first compound is a $\pi \pi \pi$ tri-radical, where all the unpaired electrons occupy $\pi$ orbitals, whereas both MM-TDB and OO-TDB are $\sigma \sigma \sigma$ tri-radicals, whose $\sigma$ orbitals bear all the unpaired electrons.

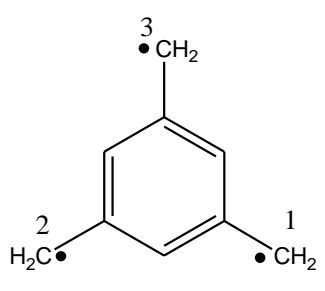

TMB

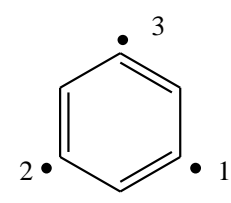

MM-TDB

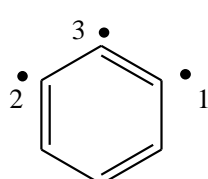

OO-TDB

Figure 1 - Structures of the three investigated tri-radicals: 1,3,5-trimethylen benzene (TMB), 1,3,5tridehydrobenzene (MM-TDB) and 1,2,3-tridehydrobenzene (OO-TDB).

TMB has been recently synthesized and characterized as having a quartet ground state.[36] Previously, only a perchlorinated derivative of TMB with quartet ground state was prepared and studied.[72] This was followed by an accurate post Hartree-Fock computational study by Kemintz, Squires and Borden,[73] showing that the tri-radical has indeed a fully-symmetric quartet ground state and that the first two doublet excited states are subject to Jahn-Teller distortion. Computational studies on related compounds can also be found in Refs. [[74, 75]]. MM-TDB is very reactive and, contrarily to its trifluoro homologue,[28] cannot be isolated in matrix, but its heat of formation in the gas phase has been determined experimentally.[76] The species has been investigated theoretically by both DFT and WF methods[74, 77] demonstrating that MM-TDB has a doublet ground state, which has a two-fold degeneracy in the $\mathrm{D}_{3 \mathrm{~h}}$ symmetry representation, but, as for TMB, undergoes Jahn-Teller distorsion to $\mathrm{C}_{2 \mathrm{v}}$. Finally, OO-TDB has been isolated in matrix and spectroscopically characterized. [78] Investigations by several post-Hartree-Fock methods[79, 80] have shown that, as found for MM-TDB, the ground state is a doublet with a pseudo-bicyclic character. Another doublet, monocyclic, is found slightly above in energy, whereas the quartet is found $2 \mathrm{eV}$ higher. For both doublets, structural parameters have also recently been determined by IVO-CASCI calculations.[81] 
Besides representing a challenging benchmark for our computational route, these three tri-radical species were chosen as they allow for a comparative study, which may help to reach a deeper understanding of the delicate relationship between their structure and the overall magnetic properties. Indeed, while TMB and MM-TDB are geometrically equivalent but with different electronic nature ( $\pi \pi \pi$ vs $\sigma \sigma \sigma)$, MM-TDB and OO-TDB have equivalent spin ground states but are geometrically different.

\section{Computational Details and Geometry Optimizations}

Geometry optimization of all species has been performed using Gaussian09[82] at the unrestricted DFT level using the hybrid B3LYP functional, in conjunction with the Dunning's correlation consistent basis set, ccpvTz, for the $\sigma \sigma \sigma$ species and the triple- $\zeta$ polarized $6-311 \mathrm{G}^{*}$ basis set for the $\pi \pi \pi \mathrm{TMB}$ system.

Optimization in $\mathrm{C}_{2 \mathrm{v}}$ symmetry for the two TDB species in a doublet spin state has been performed through a relaxed scan of the $\mathrm{C}_{1}-\mathrm{C}_{2}$ distance, to obtain both $\mathrm{A}_{1}$ and $\mathrm{B}_{2}$ states in their energy minimum and the energy profile along that coordinate.

Table 1 - Geometrical parameters for TMB, MM-TDB and OO-TDB in $\mathrm{D}_{3 \mathrm{~h}}$ and $\mathrm{C}_{2 \mathrm{v}}$. Distances are in $\AA$.

\begin{tabular}{|c|c|c|c|}
\hline $\begin{array}{l}\mathrm{r}_{13}=\mathrm{r}_{12}=\mathrm{r}_{23}=4.914 \\
\mathrm{r}_{\mathrm{ab}}=\mathrm{r}_{\mathrm{ac}}=\mathrm{r}_{\mathrm{bc}}=2.438 \\
\mathrm{r}_{\alpha \beta}=\mathrm{r}_{\alpha \gamma}=\mathrm{r}_{\beta \gamma}=2.490 \\
\mathrm{r}_{\mathrm{a} \gamma}=\mathrm{r}_{\gamma \mathrm{b}}=\mathrm{r}_{\mathrm{a} \alpha}=\mathrm{r}_{\beta \mathrm{b}}=\mathrm{r}_{\mathrm{c} \alpha}=\mathrm{r}_{\beta \mathrm{c}}=1.423\end{array}$ & $\underbrace{\mathrm{B}}_{\substack{\mathrm{c} \\
\mathrm{TMB} \mathrm{D}_{3 \mathrm{~h}}}}$ & ${\text { MM-TDB } \mathrm{D}_{3 \mathrm{~h}}}_{\mathrm{c}}^{\mathrm{b}}$ & $\begin{array}{l}\mathrm{r}_{13}=\mathrm{r}_{12}=\mathrm{r}_{23}=2.328 \\
\mathrm{r}_{\mathrm{ab}}=\mathrm{r}_{\mathrm{ac}}=\mathrm{r}_{\mathrm{bc}}=2.446 \\
\mathrm{r}_{\mathrm{a} 3}=\mathrm{r}_{\mathrm{b} 3}=\mathrm{r}_{\mathrm{a} 2}=\mathrm{r}_{\mathrm{c} 2}=\mathrm{r}_{\mathrm{c} 1}=\mathrm{r}_{\mathrm{b} 1}=1.380\end{array}$ \\
\hline $\begin{array}{l}\mathrm{r}_{12}=4.915 ; \mathrm{r}_{13}=\mathrm{r}_{23}=4.938 \\
\mathrm{r}_{\mathrm{ab}}=2.415 ; \mathrm{r}_{\mathrm{ac}}=\mathrm{r}_{\mathrm{bc}}=2.429 \\
\mathrm{r}_{\alpha \beta}=2.474 \\
\mathrm{r}_{\alpha \gamma}=r_{\beta \gamma}=2.472 \\
\mathrm{r}_{\mathrm{a} \gamma}=\mathrm{r}_{\gamma \mathrm{b}}=1.405 \\
\mathrm{r}_{\mathrm{a} \alpha}=\mathrm{r}_{\beta \mathrm{b}}=1.422 \\
\mathrm{r}_{\mathrm{c} \alpha}=\mathrm{r}_{\beta \mathrm{c}}=1.414\end{array}$ & $\overbrace{\substack{\mathrm{C} \\
\mathrm{TMB} \mathrm{C}_{2 \mathrm{v}} \mathrm{A}_{2}}}^{\mathrm{a}}$ & $\overbrace{\substack{\mathrm{C} \\
\mathrm{TMB} \mathrm{C}_{2 \mathrm{v}} \mathrm{B}_{1}}}^{\beta} \overbrace{\mathrm{CH}_{2}}^{\mathrm{a}}$ & $\begin{array}{l}r_{12}=5.034 ; r_{13}=r_{23}=4.862 \\
r_{a b}=2.395 ; r_{a c}=r_{b c}=2.427 \\
r_{\alpha \beta}=2.474 \\
r_{\alpha \gamma}=r_{\beta \gamma}=2.499 \\
r_{a \gamma}=r_{\gamma b}=1.414 \\
r_{a \alpha}=r_{\beta b}=1.419 \\
r_{c \alpha}=r_{\beta c}=1.417\end{array}$ \\
\hline $\begin{array}{l}\mathrm{r}_{12}=1.919 ; \mathrm{r}_{13}=\mathrm{r}_{23}=2.274 \\
\mathrm{r}_{\mathrm{ab}}=2.393 ; \mathrm{r}_{\mathrm{ac}}=\mathrm{r}_{\mathrm{bc}}=2.587 \\
\mathrm{r}_{\mathrm{a} 3}=\mathrm{r}_{\mathrm{b} 3}=1.396 \\
\mathrm{r}_{\mathrm{a} 2}=\mathrm{r}_{\mathrm{b} 1}=1.363 \\
\mathrm{r}_{\mathrm{c} 2}=\mathrm{r}_{\mathrm{c} 1}=1.351\end{array}$ & $\overbrace{\mathrm{c}}^{\mathrm{c}}$ & $\overbrace{\mathrm{MM}-\mathrm{TDB} \mathrm{C}_{2 \mathrm{v}} \mathrm{B}_{2}}^{\bullet_{1}^{3}}$ & $\begin{array}{l}\mathrm{r}_{12}=2.346 ; \mathrm{r}_{13}=\mathrm{r}_{23}=2.186 \\
\mathrm{r}_{\mathrm{ab}}=2.550 ; \mathrm{r}_{\mathrm{ac}}=\mathrm{r}_{\mathrm{bc}}=2.467 \\
\mathrm{r}_{\mathrm{a} 3}=\mathrm{r}_{\mathrm{b} 3}=1.355 \\
\mathrm{r}_{\mathrm{a} 2}=\mathrm{r}_{\mathrm{b} 1}=1.391 \\
\mathrm{r}_{\mathrm{c} 1}=\mathrm{r}_{\mathrm{c} 2}=1.379\end{array}$ \\
\hline $\begin{array}{l}r_{12}=1.692 ; r_{13}=r_{23}=1.342 \\
r_{a b}=2.341 ; r_{a c}=r_{b c}=1.403 \\
r_{a 2}=r_{b 1}=1.370\end{array}$ & OO-TDB $\mathrm{C}_{2 \mathrm{v}} \mathrm{A}_{1}$ & ${\mathrm{OO}-\mathrm{TDB} \mathrm{C}_{2 \mathrm{v}} \mathrm{B}_{2}}_{\mathrm{b}}^{\mathrm{a}}$ & $\begin{array}{l}\mathrm{r}_{12}=2.343 ; \mathrm{r}_{13}=\mathrm{r}_{23}=1.287 \\
\mathrm{r}_{\mathrm{ab}}=2.471 ; \mathrm{r}_{\mathrm{ac}}=\mathrm{r}_{\mathrm{bc}}=1.406 \\
\mathrm{r}_{\mathrm{a} 2}=\mathrm{r}_{\mathrm{b} 1}=1.394\end{array}$ \\
\hline
\end{tabular}

The geometrical structures optimized in the present work (see Table 1 for the geometrical parameters most significant for the discussion which will follow) are in basic agreement with those of refs. [73, 77, 79]. A 
complete set of bond lengths and angles, along with the mean value of the spin operator $\mathrm{S}^{2}$, can be found in Table S1 of the Supporting Information. In Table S1 one can notice that in few cases there can be some spin contamination for the doublet states and there are attempts to overcome that problem in geometry optimization [39]. In all cases the planarity of the skeleton is preserved so that the point group symmetry is $D_{3 h}$ (or $C_{2 v}$ for the states subjected to Jahn-Teller distortion). From the values of $r_{a b}$ and $r_{\alpha \beta}$ (for TMB) or $r_{12}$ (for TDB), it is apparent that geometrical changes accompanying the distortion in TMB are smaller than in TDB. For the former systems only slight deformations from a perfect hexagon are found and in particular the longest and shortest sides differ by about $0.02 \AA$. Furthermore the distance between the magnetic centers changes by no more than $0.05 \AA$. Conversely, for TDB significant changes in the molecular geometry are observed with respect to a regular hexagon. Moreover the distance between the magnetic centers is different for the several states of the MM-TDB system with relevant consequences on the stability of the $A_{1}$ and $B_{2}$ states. With reference to the $D_{3 h}$ geometry of the MM-TDB system, $r_{12}$ slightly increases for the $B_{2}$ doublet state and decreases significantly for the $\mathrm{A}_{1}$ state, whereas $\mathrm{r}_{13}=\mathrm{r}_{23}$ decreases in the $\mathrm{B}_{2}$ state and only slightly in $\mathrm{A}_{1}$ state.

For each molecule, the doublet-quartet gap, $\Delta_{\mathrm{DQ}}=\mathrm{E}_{(\mathrm{S}=1 / 2)}-\mathrm{E}_{(\mathrm{S}=3 / 2)}$, is evaluated according to the route described in details in Ref. [[47]] and based on DDCI scheme. Briefly, ROHF molecular orbitals (MOs) are obtained by a high spin SCF calculation carried out with GAMESS.[83] When required, magnetic MOs are localized onto the magnetic sites and virtual MOs can be modified and rearranged, exploiting the addition, through an ad hoc procedure, of supplementary point charges on the magnetic sites. The corresponding integrals are then transformed from atomic to molecular basis set. For saving computational time, the final variational CI calculation of $\Delta_{\mathrm{DQ}}$ can be performed on a reduced active MO space, and the value obtained corrected by the Complementary Space Perturbative Approach (CSPA), which takes into account the remaining MO space.

\section{Theoretical Considerations}

The essential physics underlying the quartet-vs-doublet ground state can be interpreted through a simple model Hamiltonian applied, for the present case of tri-radicals, to the minimal 3 orbital - 3 electron full configurational space $\mathrm{CAS}(3,3)$, which is obtained considering 1 orbital per magnetic site, which can be either a $\sigma$ (OO-TDB and MM-TDB) or a $\pi$ (TMB) orbital. In such a localized basis set, we can build a model Hubbard Hamiltonian including an explicit exchange term, whose general expression reads:

$$
H=\sum_{i<j, \sigma} t_{i j}\left(a_{i \sigma}^{+} a_{j \sigma}+a_{j \sigma}^{+} a_{i \sigma}\right)+\sum_{j, \sigma} \varepsilon_{j} n_{j \sigma}+\sum_{j} U_{j} n_{j \uparrow} n_{j \downarrow}+\sum_{i<j, \sigma \neq \tau} k_{i j} a_{i \sigma}^{+} a_{j \tau}^{+} a_{i \tau} a_{j \sigma}
$$

where $a_{j, \sigma}^{+}\left(a_{j, \sigma}\right)$ is the creation (annihilation) operator of one electron with spin $\sigma$ in the orbital $j$, $n_{j, \sigma}=a_{j, \sigma}^{+} a_{j, \sigma}$ is the number operator. The parameter $t$ is the resonance, or hopping, integral, $\varepsilon$ is the site orbital energy, $U$ the on-site Coulomb repulsion and $k$ the effective exchange; $t$ is always negative and its value measures the stabilization accompanying electron delocalization. At the same time, $U$ destabilizes 
orbital double occupancy and $k$ introduces the intrinsic magnetic properties. For the species with three equivalent magnetic sites, such as TMB and MM-TDB in their $\mathrm{D}_{3 \mathrm{~h}}$ form, eq. (1) reduces to:

$$
H=t \sum_{j, \sigma}\left(a_{j, \sigma}^{+} a_{j+1, \sigma}+a_{j+1, \sigma}^{+} a_{j, \sigma}\right)+\varepsilon \sum_{j, \sigma} n_{j, \sigma}+U \sum_{j} n_{j \uparrow} n_{j \downarrow}+k \sum_{i<j, \sigma \neq \tau} a_{i, \sigma}^{+} a_{j, \tau}^{+} a_{i, \tau} a_{j, \sigma}
$$

where the first summation is cyclic with site 4 coincident with site 1 . This does not hold for OO-TDB and for the $\mathrm{C}_{2 \mathrm{v}}$ forms of MM-TDB arising from Jahn-Teller distortion. In such cases, site 1 and 2 are equivalent but site 3 is different (see Fig. 1), and the fact that the three orbitals may be on non-equivalent magnetic sites should be taken into account. Consequently, Eq. (1) can be written as:

$$
\begin{aligned}
& H=t \sum_{j=1,2 ; \sigma}\left(a_{j, \sigma}^{+} a_{3, \sigma}+a_{3, \sigma}^{+} a_{j, \sigma}\right)+t_{1} \sum_{j \neq k=1,2 ; \sigma} a_{j, \sigma}^{+} a_{k, \sigma}+\sum_{j, \sigma} \varepsilon_{j} n_{j, \sigma}+\sum_{j} U_{j} n_{j \uparrow} n_{j \downarrow}+ \\
& +\sum_{\sigma \neq \tau}\left(k_{1} a_{1, \sigma}^{+} a_{2, \tau}^{+} a_{1, \tau} a_{2, \sigma}+k a_{1, \sigma}^{+} a_{3, \tau}^{+} a_{1, \tau} a_{3, \sigma}+k a_{2, \sigma}^{+} a_{3, \tau}^{+} a_{2 \tau} a_{3, \sigma}\right)
\end{aligned}
$$

where $t$ and $k$ are the hopping and the exchange contribution, respectively when magnetic site 3 is involved; $t_{1}$ is the direct 1-2 hopping and $k_{1}$ is the effective exchange involving sites 1 and 2.

For the 3-electron-3-orbital case and considering the configurations with $S_{z}=1 / 2$, we can write, for both the $D_{3 h}$ and $\mathrm{C}_{2 \mathrm{v}}$ point groups, 9x9 matrix representations of the above Hubbard Hamiltonian (eqns. 2 and 3), which are reported in symbolic form in the Table S2 of Supporting Information. An analysis at a semi-quantitative level of these matrices may yield useful information on the tri-radical systems under study, and may help to rationalize the relevant physical features which push the system toward a low or high spin ground state.

First of all, we can see that in the limit of very small hopping $\left(t, t_{1} \rightarrow 0\right)$, the two matrices can be transformed and factorized in two sub-matrices: one at high energy for the configurations with double occupancies of the orbital, the other, at low energy, for the three configurations with 1 electron per magnetic site (see central row of Table S2). For $\mathrm{D}_{3 \mathrm{~h}}$ we have then the quartet at energy $-2 k$ and the two degenerate doublets at energy $k$, while the lowest doublets with double occupancy are found at energy $U-k$, which is always expected to be higher than $k$. For $\mathrm{C}_{2 \mathrm{v}}$ we are faced with a slightly more complex situation due to the differences between one site with respect to the other two (see central row Table S2). However, we can make the same considerations concerning the quartet $v s$ doublet ground state.

In addition, we can see that the degeneracy of the two lowest doublets is now removed. In summary, in the limit of small hopping, the three lowest states are those with single occupancy in the magnetic sites and the ground state is always the quartet, in accordance with the Hund's rule.

Increasing the hopping, i.e. the electron delocalization, we observe rather different situations. The matrix for $\mathrm{D}_{3 \mathrm{~h}}$, transformed to separate the quartet from the doublets, is explicitly reported in the last row of Table $\mathrm{S} 2$ for the interested reader. On the contrary, the complete Hamiltonian matrix for $\mathrm{C}_{2} \mathrm{v}$ symmetry is far too complex to be straightforwardly displayed. It is clear that, while the quartet is still decoupled from the other eight eigenstates, the same does not hold any more for the doublet states with single occupancy. There, as a result of 
the contamination of the configurations with double occupied magnetic sites, the energy of the doublets may go below that of the quartet.

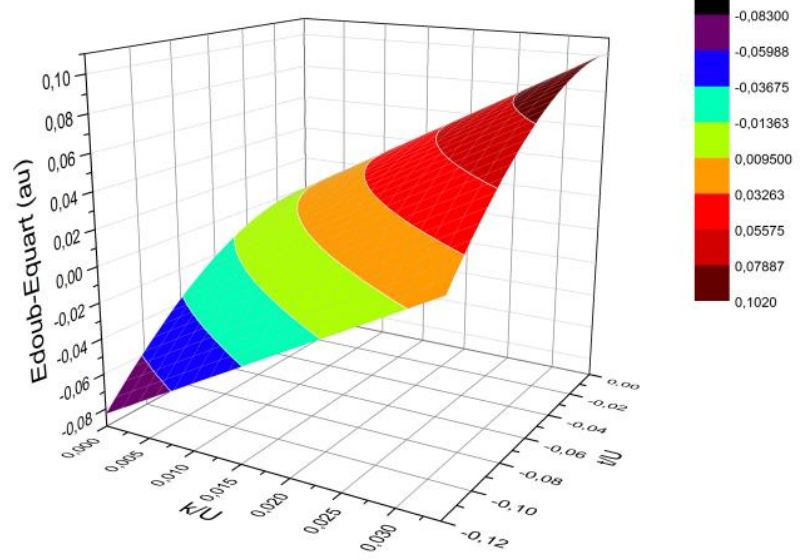

Figure 2 - 3D plot of the energy difference between the two-fold degenerate (in $\mathrm{D}_{3 \mathrm{~h}}$ ) doublet and the quartet as a function of the effective exchange $k$ and the hopping $t$. Both parameters are in unit of the onsite Coulomb repulsion $U$.

This concept should be clarified by Figure 2, where for $D_{3 h}$ the energy difference between the lowest degenerate doublet and the quartet is reported as a function of the effective exchange $(k)$ and the hopping $(t)$ in units of the on-site Coulomb repulsion (U). For a given value of $\mathrm{k} / \mathrm{U}$, the ground state is the quartet for small values of $t / U$ and becomes the doublet as the hopping increases. This indeed occurs since the doublet eigenstates increase their double occupation character on magnetic sites, as a consequence of the increased electronic delocalization. The eigenstates with strong double occupation character are always found at higher energies.

The above discussion based on the Hubbard Hamiltonian may be exploited in order to compare the different behavior of the two $\mathrm{D}_{3 \mathrm{~h}}$ species TMB e MM-TDB. In fact, despite the topology of the two systems is the same, the $\pi \pi \pi$ species is found to have a quartet ground state, while the $\sigma \sigma \sigma$ one a doublet ground state.[73, 77, 78] Although it is known that the doublet state shows a two-fold degeneracy in $\mathrm{D}_{3 \mathrm{~h}}$ and undergoes Jahn-Teller distortions, a useful comparison can be done in $\mathrm{D}_{3 \mathrm{~h}}$ point group using the corresponding matrix of Table S2. According to this approximate treatment, we expect that the Hubbard Hamiltonian for TMB has a larger exchange and a smaller hopping than MM-TDB. An effective estimate of the value of the parameters entering the Hubbard Hamiltonian can be done by exploiting a CI calculation in the same CASCI(3,3) space. This can be done by minimizing the discrete functional (corresponding to a least squares fitting):

$$
I=\sum_{i=1}^{9} w_{i}\left[E_{i}(C A S C I)-E_{i}(t, k, U)\right]^{2}
$$

where $E_{i}(C A S C I)$ and $E_{i}(t, k, U)$ are the eigenvalues of the CASCI calculation and those of the Hubbard Hamiltonian, respectively, and we have verified the correspondence of the eigenfunctions. The weights $w_{i}$ are 
chosen inversely proportional to the excitation energy. In such a way the three parameters $t, k, U$ are those that represent at best the true Hamiltonian matrix, although the latter includes also some minor contributions which are not present in the (simplified) Hubbard operator. The results of such a fitting are reported in Table 2.

As expected, TMB has a smaller hopping and a larger exchange than MM-TDB and despite the U value computed for the $\sigma \sigma \sigma$ system is about twice the one found for $\pi \pi \pi$ one, the differences of $\mathrm{t}$ and $\mathrm{k}$ are sufficient to obtain a doublet ground state. The doublet-quartet gap $\Delta_{\mathrm{DQ}}$, computed with this model is practically the same obtained with CASCI in the minimal space. Furthermore, the relative position of the other doublet states is also well reproduced by the simple model. Yet, these values are qualitative estimates, but only accurate calculations in a much larger configurational space may give quantitative values for the gap.

It should also be noticed that the results of the fitting show that the on-site Coulomb repulsion $U$ is much larger than the delocalization term $t$. This means that for the present case one might also exploits a perturbative treatment for the two lowest doublet states, which at the zero order are linear combinations of the three configurations with single occupation (eigenstates of the $3 \times 3$ matrix of Table S2), while the quartet is always decoupled. Indeed, the doublet-quartet gap for TMB and MM-TDB of Table 2, which is $3 k$ for $t=0$, results 0.027 instead of 0.033 for TMB and -0.019 instead of 0.006 for MM-TDB, respectively.

Table 2 - Parameters of the Hamiltonian of Eq.1 ( $\mathrm{D}_{3 \mathrm{~h}}$ point group) obtained by a fitting of the eigenvalues of the $\operatorname{CASCI}(3,3)$ calculation, eq. (4). $\Delta(i)=E_{i}-E_{0}$ are the energy differences between the $i$-th state and the ground state, in Hartree. The ground state is the quartet for TMB and the degenerate doublet for MM-TDB. The exited states are all doublets except the first excited state for MM-TDB (corresponding to $\Delta(2)$ ) that is the quartet.

\begin{tabular}{|c|c|c|}
\hline Parameter & TMB D $_{3 \mathrm{~h}}$ & MM-TDB D \\
$3 \mathrm{~h}$ \\
\hline$t$ & -0.017 & -0.042 \\
\hline$U$ & 0.244 & 0.416 \\
\hline$k$ & 0.011 & 0.002 \\
\hline$\Delta(1) \mathrm{CI} /$ Model & $0.027 / 0.027$ & $0 / 0$ \\
\hline$\Delta(2) \mathrm{CI} /$ Model & $0.027 / 0.027$ & $0.019 / 0.019$ \\
\hline$\Delta(3) \mathrm{CI} /$ Model & $0.236 / 0.237$ & $0.379 / 0.379$ \\
\hline$\Delta(4) \mathrm{CI} /$ Model & $0.236 / 0.237$ & $0.379 / 0.379$ \\
\hline$\Delta(5) \mathrm{CI} /$ Model & $0.244 / 0.255$ & $0.430 / 0.436$ \\
\hline$\Delta(6) \mathrm{CI} /$ Model & $0.295 / 0.303$ & $0.448 / 0.440$ \\
\hline$\Delta(7) \mathrm{CI} /$ Model & $0.295 / 0.303$ & $0.522 / 0.523$ \\
\hline$\Delta(8) \mathrm{CI} /$ Model & $0.307 / 0.278$ & $0.522 / 0.523$ \\
\hline
\end{tabular}

The species in $\mathrm{C}_{2 \mathrm{v}}$ point group do not allow for a confident fitting, because the number of parameters (8) equals the number of reference data ( 8 energy differences). Nonetheless, the value of the diagonal term $\mathrm{U}$ obtained with the $\mathrm{D}_{3 \mathrm{~h}}$ fitting can be bi-univocally mapped to the diagonal terms of the CASCI matrix, and further rescaled to set at 0 the lowest term (see Table S3 in the Supporting Information). A similar procedure could be thus adopted for $\mathrm{C}_{2} \mathrm{v}$, using the diagonal terms of the matrixes of Table S3 and fitting the remaining parameters with the CASCI energy differences obtained at the optimized geometry. For OO-TDB, we performed the fitting of the parameters only for the $\mathrm{B}_{2}$ state, since at the equilibrium geometry of the $\mathrm{A}_{1}$ state the quartet, as it will be shown in the next section, is very high in energy with several different eigenstates in between. The values obtained are reported in Table 3. 
Comparing Table 2 and Table 3, we can see that the hopping for the $\sigma \sigma \sigma$ species appears more sensitive to the geometrical distortions than for the $\pi \pi \pi$ species. This is a consequence of the different deformation, which is larger for TDBs (see Table 1), as well as of the different impact of the geometrical changes in the two systems: in TMB the centroids of the magnetic orbitals are more distant than in TDB, and this is also reflected by the smaller value of $t$. Indeed, for TMB the ground state is the quartet even in $\mathrm{C}_{2 \mathrm{v}} . U$, as expected, is found larger in the $\sigma \sigma \sigma$ system TDB than in TMB. The value for the different site $3\left(U_{3}\right)$ increases for TMB and for MMTDB $\mathrm{A}_{1}$ but decreases for MM-TDB $\mathrm{B}_{2}$. The exchange $k$ decreases in TMB and does not change in TDB, $k_{1}$ behaves like $k$ for TMB but in MM-TDB becomes negligible and this indicates that the configuration with antiparallel spins in the sites 1 and 2 is favored. The different values of the hopping find an explanation in the geometrical changes occurring in the two species when going from $D_{3 h}$ to $C_{2 v}$.

For the OO-TDB system in the $\mathrm{B}_{2}$ state, we can make a comparison with its MM analog. We can see that $\mathrm{r}_{12}$ is about the same in the two species and this is reflected by the value of $t_{1}$ which is about the same. The $\mathrm{r}_{13}=\mathrm{r}_{23}$ distances are smaller in OO-TDB than in MM-TDB and $t$ is then found larger in the first case. The $U$ term is about the same in both systems, while $U_{3}$ is smaller in the OO species. This should indicate that double occupation in the magnetic orbital of site 3 is less disfavored than in the MM analog, this conclusion being confirmed by the composition of the CASCI eigenstates. Again, the exchange involving the site 1 and 2 vanishes.

Table 3 - Parameters of the Hamiltonian of eq. $3\left(\mathrm{C}_{2 \mathrm{v}}\right.$ point group) obtained by a fitting of the eigenvalues of the $\operatorname{CASCI}(3,3)$ calculation, eq. (4). $\Delta(i)=E_{i}-E_{0}$ are the energy differences between the $i$-th state and the ground state, in Hartree. $\varepsilon, \varepsilon_{3}, U$ and $U_{3}$ are obtained analytically from the diagonal terms of Table S2. The ground state is the quartet for TMB and the lowest doublet for the other species. The exited states are all doublets except the second excited state for MM-TDB and OOTDB (corresponding to $\Delta(3)$ ) that is the quartet.

\begin{tabular}{|c|c|c|c|c|c|}
\hline Parameter & TMB A $_{2}$ & TMB B $_{1}$ & MM-TDB $_{1}$ & MM-TDB B $_{2}$ & OO-TDB B $_{2}$ \\
\hline$t$ & -0.015 & -0.020 & -0.042 & -0.054 & -0.071 \\
\hline$t_{l}$ & -0.017 & -0.006 & -0.084 & -0.034 & -0.030 \\
\hline$k$ & 0.008 & 0.010 & 0.003 & 0.003 & 0.006 \\
\hline$k_{l}$ & 0.007 & 0.006 & 0.000 & 0.000 & 0.000 \\
\hline$\varepsilon$ & -0.123 & -0.124 & -0.187 & -0.184 & -0.153 \\
\hline$\varepsilon_{3}$ & -0.137 & -0.127 & -0.300 & -0.128 & 0.004 \\
\hline$U$ & 0.264 & 0.265 & 0.348 & 0.414 & 0.434 \\
\hline$U_{3}$ & 0.293 & 0.270 & 0.440 & 0.307 & 0.192 \\
\hline$\Delta(1) \mathrm{CI} /$ Model & $0.017 / 0.018$ & $0.022 / 0.022$ & $0.056 / 0.056$ & $0.014 / 0.014$ & $0.039 / 0.039$ \\
\hline$\Delta(2) \mathrm{CI} /$ Model & $0.020 / 0.020$ & $0.022 / 0.022$ & $0.074 / 0.074$ & $0.035 / 0.035$ & $0.067 / 0.067$ \\
\hline$\Delta(3) \mathrm{CI} /$ Model & $0.255 / 0.261$ & $0.248 / 0.253$ & $0.323 / 0.327$ & $0.357 / 0.359$ & $0.329 / 0.328$ \\
\hline$\Delta(4) \mathrm{CI} /$ Model & $0.261 / 0.265$ & $0.251 / 0.260$ & $0.392 / 0.394$ & $0.367 / 0.367$ & $0.344 / 0.344$ \\
\hline$\Delta(5) \mathrm{CI} /$ Model & $0.264 / 0.283$ & $0.262 / 9.275$ & $0.477 / 466$ & $0.437 / 0.440$ & $0.425 / 0.426$ \\
\hline$\Delta(6) \mathrm{CI} /$ Model & $0.303 / 0.297$ & $0.304 / 0.299$ & $0.477 / 0.478$ & $0.451 / 0.448$ & $0,430 / 0.434$ \\
\hline$\Delta(7) \mathrm{CI} /$ Model & $0.314 / 0.322$ & $0.305 / 0.311$ & $0.536 / 0.534$ & $0.525 / 0.524$ & $0.572 / 0.572$ \\
\hline$\Delta(8) \mathrm{CI} /$ Model & $0.321 / 0.325$ & $0.319 / 0.321$ & $0.640 / 0.643$ & $0.556 / 0.559$ & $0.597 / 0.595$ \\
\hline
\end{tabular}

In general terms the energy position of the nine states is well reproduced by the model. The agreement for the double-quartet energy gap, which corresponds in the two tables to $\Delta(1)$ for TMB and $\Delta(2)$ for MM-TDB and OO-TDB, is always satisfactory, but we underline again that a quantitative evaluation of the gap requires 
much more extensive calculations, although the simple model and the CASCI in the minimal space, already give an indication of whether or not the ground state is the quartet.

\section{DDCI Calculations}

Although the theoretical considerations discussed above give an interpretative overview of the basic physics underlying the $\sigma \sigma \sigma$ and $\pi \pi \pi$ tri-radical species here considered, a quantitative evaluation of the energy gaps between their lowest eigenstates requires more accurate calculations. Since these species are quite small, as mentioned in the Introduction many WF state-of-the-art methods can be considered with confidence. Here we have chosen DDCI, in the recent implementation of ref. [47].

Once a basis set of Molecular Orbitals (MOs) has been obtained, DDCI philosophy consists in adding single and double excitations, involving at least one of the magnetic orbitals to the minimal $\operatorname{CASCI}(3,3)$. The whole space can be divided into 8 classes, summarized in Table 4 and characterized by different level of excitation between the three partitions of the MOs (double occupied, magnetic and empty or virtual MOs).

Table 4 - Classes of excitation in DDCI for 3-electrons in 3 magnetic sites systems arising from $\mathrm{CAS}(3,3)$. In our notation $\mathrm{n}, \mathrm{m}, \mathrm{l}$ indicates the class with $\mathrm{n}$ electrons in the double occupied (inactive) MOs, $\mathrm{m}$ electrons in the 3 magnetic orbitals and 1 electrons in the empty (external) MOs. $\mathrm{N}$ is the number of core electrons filling the inactive orbitals of the $\operatorname{CASCI}(3,3)$. In the last column we report the notation of ref.[84] but we keep using the A, B, etc. notation in the text for simplicity.

\begin{tabular}{|l|l|l|l|}
\hline Class & Description & & {$[84]$} \\
\hline $\mathrm{N}, 3,0$ & the minimal CAS(3,3) space & A & \\
\hline $\mathrm{N}, 2,1$ & magnetic-to-empty & B & $1 \mathrm{p}$ \\
\hline $\mathrm{N}, 1,2$ & double magnetic-to-empty & $\mathrm{C}$ & $2 \mathrm{p}$ \\
\hline $\mathrm{N}-1,4,0$ & occupied-to-magnetic & $\mathrm{D}$ & $1 \mathrm{~h}$ \\
\hline $\mathrm{N}-1,3,1$ & $\begin{array}{l}\text { occupied-to-empty } \\
\text { occupied-to-magnetic+magnetic-to-empty }\end{array}$ & $\mathrm{E}$ & $1 \mathrm{~h} 1 \mathrm{p}$ \\
\hline $\mathrm{N}-1,2,2$ & occupied-to-empty+magnetic-to-empty & $\mathrm{F}$ & $1 \mathrm{~h} 2 \mathrm{p}$ \\
\hline $\mathrm{N}-2,5,0$ & double occupied-to-magnetic & $\mathrm{G}$ & $2 \mathrm{~h}$ \\
\hline $\mathrm{N}-2,4,1$ & occupied-to-magnetic+occupied-to-empty & $\mathrm{H}$ & $2 \mathrm{~h} 1 \mathrm{p}$ \\
\hline
\end{tabular}

\section{a) 1,3,5-trimetylenbenzene (TMB)}

The relevant interatomic distances for the optimized quartet in $\mathrm{D}_{3 \mathrm{~h}}$ point group and doublets in $\mathrm{C}_{2 \mathrm{v}}$ are reported in Table 1, in agreement with those of ref. [73]. Jahn-Teller distorsions do not cause large changes in the geometry.

DDCI calculations have been performed at all geometries. Among the MOs obtained for the quartet at ROHF/6-31G* level using GAMESS,[83] singly occupied (magnetic) ones have been localized [47] in the magnetic sites (Figure 3) and the 1s orbitals of the carbon atoms are kept frozen in the excitations. In the quartet state, the MOs space is then made by 21 double occupied, 3 singly occupied and 120 virtual. The space can be partitioned to have a set where the Hamiltonian is diagonalized (variational), while the corrections due the remaining set are included by second order Möller-Plesset perturbation (Complementary Active 
Perturbative Approach, CSPA).[47] The extent of convergence found with CSPA in the present case is reported in the Supplementary Information.

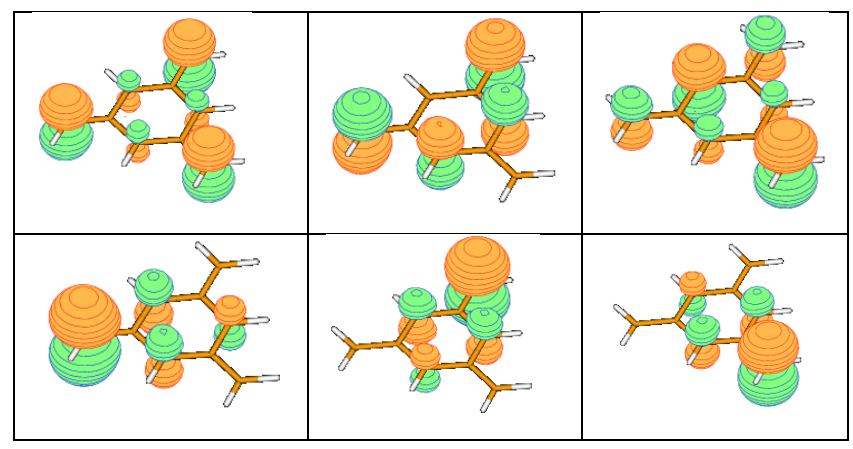

Figure 3 - Canonical (upper row) and localized (lower row) singly occupied MOs for the quartet ground state of TMB in $\mathrm{D}_{3} \mathrm{~h}$.

Table 5 reports the values obtained with a full variational calculation where all the empty MOs are considered virtually active, hence included in the variational space.

Table 5 -DDCI energy differences between the two excited doublets and the quartet ground state for TMB computed using localized orbitals and with 120 virtually active MOs (full variational). The Adiabatic values are obtained as the difference between the $\mathrm{C}_{2 \mathrm{v}}$ doublets and the $\mathrm{D}_{3 \mathrm{~h}}$ quartet each at its equilibrium geometry.

\begin{tabular}{|c|c|c|c|c|}
\hline & $\Delta \mathrm{E}_{1}\left(\mathrm{~cm}^{-1}\right)$ & $\Delta \mathrm{E}_{2}\left(\mathrm{~cm}^{-1}\right)$ & $\Delta \mathrm{E}_{1}\left(\mathrm{~cm}^{-1}\right)^{\mathrm{a}}$ & $\Delta \mathrm{E}_{2}\left(\mathrm{~cm}^{-1}\right)^{\mathrm{a}}$ \\
\hline $\mathrm{D}_{3} \mathrm{~h}$ & 5085 & 5086 & 6394 & 6394 \\
\hline $\mathrm{C}_{2} \mathrm{~V} \mathrm{~A}_{2}$ & 3931 & 4441 & - & - \\
\hline $\mathrm{C}_{2} \mathrm{~V} \mathrm{~B}_{1}$ & 4467 & 4598 & - & - \\
\hline Adiabatic & 4493 & 6047 & $5486\left(4822^{\mathrm{b}} ; 5135^{\mathrm{c}}\right)$ & $5556\left(4787^{\mathrm{b}} ; 5764^{\mathrm{c}}\right)$ \\
\hline
\end{tabular}

${ }^{\mathrm{a}} \mathrm{CASSCF}$ results of Ref. [73]; ${ }^{\mathrm{b}} \mathrm{CASPT} 2 \mathrm{~N}$ results of Ref. [73]; ${ }^{\mathrm{c}} \mathrm{CI}$ results of Ref. [73]

In $\mathrm{D}_{3 \mathrm{~h}}$ the two doublet states are degenerate and the ground state is a quartet, as also confirmed experimentally [36]. DDCI finds the doublet-quartet gap at the $\mathrm{D}_{3 \mathrm{~h}}$ geometry $\sim 1300 \mathrm{~cm}^{-1}$ lower that the CASSCF value of Ref.[73].

At the $\mathrm{C}_{2 \mathrm{v}}$ geometry, the ground state is still the quartet but the Jahn-Teller splitting breaks the degeneracy of the $A_{2}$ and $B_{1}$ doublets, lowering the gap by 1154 and $645 \mathrm{~cm}^{-1}$ at the $A_{2}$ geometry, and by 618 and $488 \mathrm{~cm}^{-1}$ at the $\mathrm{B}_{2}$ geometry, respectively. The lowest doublet has always $\mathrm{A}_{2}$ symmetry, in agreement with the CI results of ref.[73] but at variance with the CASSCF and CASPT2N results reported in the same reference. The energy difference between the quartet at the $\mathrm{C}_{2 \mathrm{v}}$ and $\mathrm{D}_{3 \mathrm{~h}}$ geometries, separately computed at CI-MRPT2 level, is 666 $\mathrm{cm}^{-1}$ at the $\mathrm{A}_{2}$ geometry and $1449 \mathrm{~cm}^{-1}$ at the $\mathrm{B}_{1}$ geometry, thus the adiabatic energy differences between the $\mathrm{C}_{2 \mathrm{v}}$ doublets and the $\mathrm{D}_{3 \mathrm{~h}}$ quartet are $4493\left(\mathrm{~A}_{2}\right)$ and $6047\left(\mathrm{~B}_{1}\right) \mathrm{cm}^{-1}$ (see Table 5), in line with previous computational [73] and experimental[36] results.

The contributions of the different classes of excitations, described in Table 4 , to the minimal CASCI $(3,3)$ space can be evaluated by performing separate DDCI calculations. In Table 6 are shown the results obtained at the $\mathrm{D}_{3 \mathrm{~h}}$ geometry, while those obtained at the $\mathrm{C}_{2 \mathrm{v}}-\mathrm{A}_{2}$ geometry, which show an analogous trend, are reported in the Supplementary Material. 
Table 6 - Contribution of the various classes of Table 4 to the minimal CASCI(3,3) (A) space. Energy differences between the two excited doublets and the quartet ground state for TMB at the $\mathrm{D}_{3 \mathrm{~h}}$ geometry, computed using localized orbitals and with 120 virtually active MOs (full variational). $\Delta \mathrm{E}_{0}$ is the difference between the ground state energies computed in the $\mathrm{AX}(\mathrm{X}=\mathrm{B}, \mathrm{H})$ and the minimal space $\mathrm{A}$. $\Delta \mathrm{E}=\Delta \mathrm{E}_{1}=\Delta \mathrm{E}_{2}$ is the degenerate doublet-quartet energy gap.

\begin{tabular}{|l|r|r|r|r|}
\hline \multicolumn{2}{|c|}{ Space } & Dimension & $\Delta \mathrm{E}_{0}\left(\mathrm{~cm}^{-1}\right)$ & $\Delta \mathrm{E}\left(\mathrm{cm}^{-1}\right)$ \\
\hline A & CAS $(3,3)$ & 9 & - & 5894 \\
\hline AB & $\mathrm{CAS}(3,3)+1 \mathrm{p}$ & 1449 & 0 & 4467 \\
\hline AC & $\mathrm{CAS}(3,3)+2 \mathrm{p}$ & 64629 & -105 & 5313 \\
\hline $\mathrm{AD}$ & $\mathrm{CAS}(3,3)+1 \mathrm{~h}$ & 261 & 0 & 4258 \\
\hline $\mathrm{AE}$ & $\mathrm{CAS}(3,3)+1 \mathrm{~h} 1 \mathrm{p}$ & 70569 & -13893 & 8119 \\
\hline $\mathrm{AF}$ & $\mathrm{CAS}(3,3)+1 \mathrm{~h} 2 \mathrm{p}$ & 5877909 & -33264 & 6197 \\
\hline $\mathrm{AG}$ & $\mathrm{CAS}(3,3)+2 \mathrm{~h}$ & 1962 & -69 & 5486 \\
\hline $\mathrm{AH}$ & $\mathrm{CAS}(3,3)+2 \mathrm{~h} 1 \mathrm{p}$ & 1013049 & -16377 & 6135 \\
\hline
\end{tabular}

The CASCI $(3,3)$ energy gap of the two doublets $\left(5894 \mathrm{~cm}^{-1}\right)$ is overestimated with respect to the full DDCI values of Table $5\left(5085 \mathrm{~cm}^{-1}\right)$. The gap is significantly lowered by the contribution of the B and D classes (see Table 6), which account for single excitations involving explicitly the magnetic orbitals, while the E class, that also includes double excitations, has an opposite effect. Also the double excitations from the minimal CASCI $(3,3)$ space, resulting from the subtraction or addition of two electrons in the magnetic orbitals (classes $\mathrm{C}$ and G, Table 6) cause a decrease of the gaps, but the final estimates are still above the full DDCI values. On the contrary, the double excitations considered in classes $\mathrm{F}$ and $\mathrm{H}$ produce small increases of both gaps. In summary, the main contributions to the increases of the gaps are provided by higher correlation and spin polarization of the quartet ground state with respect to that of the doublets, as witnessed by the $\mathrm{E}, \mathrm{F}$ and $\mathrm{H}$ classes of excitations. Notice that in the analysis of Table 6, the effect of interference between the various classes, which is the reason because the final values of Table 5 are rather different from that of Table 6 and makes quantitatively unreliable the use of second order perturbation to the CAS(3,3), is missing. A full investigation of the combined effect of the classes of excitation would require a large number of extensive calculations and we have thus limited our analysis to the effect of the addition of each single class to the minimal CAS $(3,3)$ space.

\section{b) 1,3,5-tridehydrobenzene (MM-TDB)}

Relevant interatomic distances for the optimized geometries in $\mathrm{D}_{3 \mathrm{~h}}$ and $\mathrm{C}_{2 \mathrm{v}}$ are reported in Table 1 and are in agreement with those of refs.[74, 77, 79]. For this $\sigma \sigma \sigma$ system, Jahn-Teller distortions modify the molecular geometry of the two $\mathrm{A}_{1}$ and $\mathrm{B}_{2}$ doublet states, which show quite different equilibrium geometries. The most significant difference occurs for the two equivalent magnetic Carbons which are very close to each other in the $\mathrm{A}_{1}$ state.

DDCI calculations have been performed along the profile of $r_{12}$ for both the $A_{1}$ and $B_{2}$ states, at geometries obtained by relaxed scans. As in the previous case, MOs have been obtained from the HF calculation of the quartet state with a ROHF/6-311G* basis set. Singly occupied (magnetic) MOs have then been localized [47] on the magnetic sites (Figure 4). The shape of the MOs in the $\mathrm{C}_{2 \mathrm{v}}$ geometries is only slightly changed in the distribution of the electronic density but is basically the same for $D_{3 h}$. As usual, the 1s orbitals of the carbon 
atoms are kept frozen in the excitations. The one electron space is then made by 12 double occupied, 3 singly occupied and 112 virtual MOs. For the present case we have considered the full MO space without any partition, which would have required CSPA corrections.

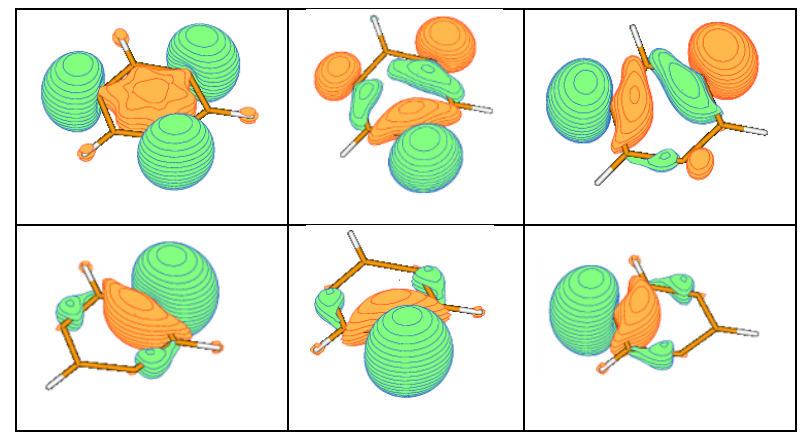

Figure 4 - Canonical (upper row) and localized (lower row) singly occupied MOs for the quartet ground state of MM-TDB in $\mathrm{D}_{3} \mathrm{~h}$.

Since, on the one hand, DDCI is known to be appropriate for the calculation of energy differences but not for their absolute values and, on the other hand, we are interested in the energy profile along the $\mathrm{r}_{12}$ coordinate, we decided to sum the DDCI energy differences to the UB3LYP energy of the ground state, as displayed in Figure 5. The full DDCI data can be found in the Supporting Information.

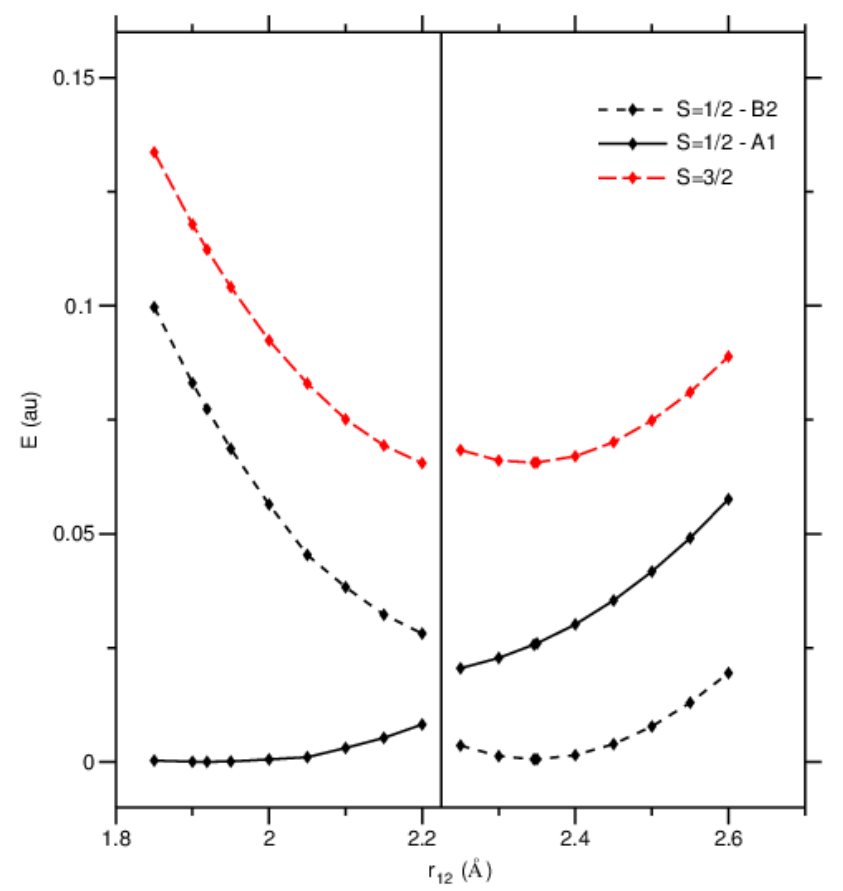

Figure 5 - Energy of the quartet and of the two doublet states of MM-TDB along the $r_{12}$ coordinate.

The energy minima in the doublet ground state are found at 1.919 and $2.346 \AA$, respectively for $\mathrm{A}_{1}$ and $\mathrm{B}_{2}$ (see Table 1). The vertical line indicates the crossing of the two states and the apparent discontinuity of each curve in crossing this line is due to the fact that the left and right region of the curve are optimized for the different states with different symmetries. 
The vertical quartet-doublet energy gap is $3.06 \mathrm{eV}\left(24655 \mathrm{~cm}^{-1}\right)$ at the $\mathrm{A}_{1}$ minimum and $1.77 \mathrm{eV}\left(14275 \mathrm{~cm}^{-1}\right)$ at the $\mathrm{B}_{2}$ minimum, while the vertical doublet-doublet (ground) gap is $2.10 \mathrm{eV}\left(16975 \mathrm{~cm}^{-1}\right)$ for the $\mathrm{A}_{1}$ ground state and $0.68 \mathrm{eV}\left(5519 \mathrm{~cm}^{-1}\right)$ for the $\mathrm{B}_{2}$ ground state. The vertical quartet-doublet $\mathrm{A}_{1}$ gap was found 68 $\mathrm{kcal} / \mathrm{mol}\left(23780 \mathrm{~cm}^{-1}\right)$ by MRMP2. [74] At the minimum of the quartet in $\mathrm{D}_{3 \mathrm{~h}}$, the gap with the two degenerate doublets, which are still the ground state, is found by DDCI at $1.04 \mathrm{eV}\left(8408 \mathrm{~cm}^{-1}\right)$ and it was found by Slipchenko and Krylov [77] at 0.726, 0719, $0726 \mathrm{eV}$, respectively by MCSCF/6-311G**, MCQPPT2/6$311 \mathrm{G}^{* *}$ and SFCCSD/6-311G**.

With respect to the $\pi \pi \pi$ TMB system, we obtain then a reversed behavior with a very stable doublet ground state and strong Jahn-Teller splitting of the two doublets, which are instead much closer in energy in TMB. This is clearly due to the stronger interaction occurring between the magnetic MOs in the $\sigma \sigma \sigma$ species with respect to the $\pi \pi \pi$ one, already underlined in the model calculations of the previous section.

\section{c) 1,2,3-tridehydrobenzene (OO-TDB)}

All the relevant interatomic distances for the OO-TDB optimized geometries in $\mathrm{C}_{2 \mathrm{v}}$ are also reported in Table 1 and are in agreement with those of refs.[78-80] As for the other $\sigma \sigma \sigma$ system, MM-DTB, Jahn-Teller distorsions are significant and the two $\mathrm{A}_{1}$ and $\mathrm{B}_{2}$ doublet states show rather different equilibrium geometries. For the $\mathrm{A}_{1}$ state the two equivalent magnetic carbon atoms are even closer than in MM-TDB (1.7 vs $1.9 \AA$ ) so that, as pointed out by Koziol et al [80], we observe a sort of incipient bond with a pseudo-double ring species for the $\mathrm{A}_{1}$ state, due to the 1-2 bonding nature of the half occupied $\mathrm{a}_{1}$ orbital.

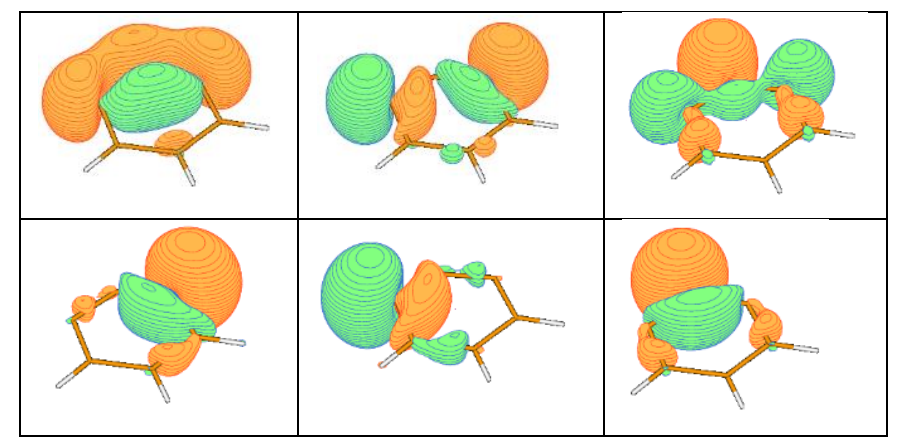

Figure 6 - Canonical (upper row) and localized (lower row) singly occupied MOs for the quartet ground state of OO-TDB at the optimized $\mathrm{B}_{2}$ geometry.

Localized and delocalized magnetic MOs, obtained at ROHF/6-311G* level, are shown in Figure 6. The MOs space is obviously identical to that of MM-TDB discussed above.

Figure 7 shows the lowest 9 eigenstates obtained with the same procedure used for MM-TDB, namely by adding the energy differences obtained by a full variational DDCI calculation to the DFT/UB3LYP ground state energy. The full DDCI data can be found in the Supporting Information. In the figure the different colors identify the energy order of the DDCI results (Sn), since there are many crossings (and avoided crossings), well visible in Figure 7, above the two magnetic doublets. The doublets keep the same order found for MM- 
TDB and indeed in the left side of the figure $\mathrm{S} 1\left(\mathrm{~A}_{1}\right)$ is the $\mathrm{B}_{2}$ state and in the right side $\mathrm{S} 1\left(\mathrm{~B}_{2}\right)$ is the $\mathrm{A}_{1}$ state. $\mathrm{S} 2$ is the $\sigma$ quartet only for $\mathrm{r}_{12}>1.95 \AA$.
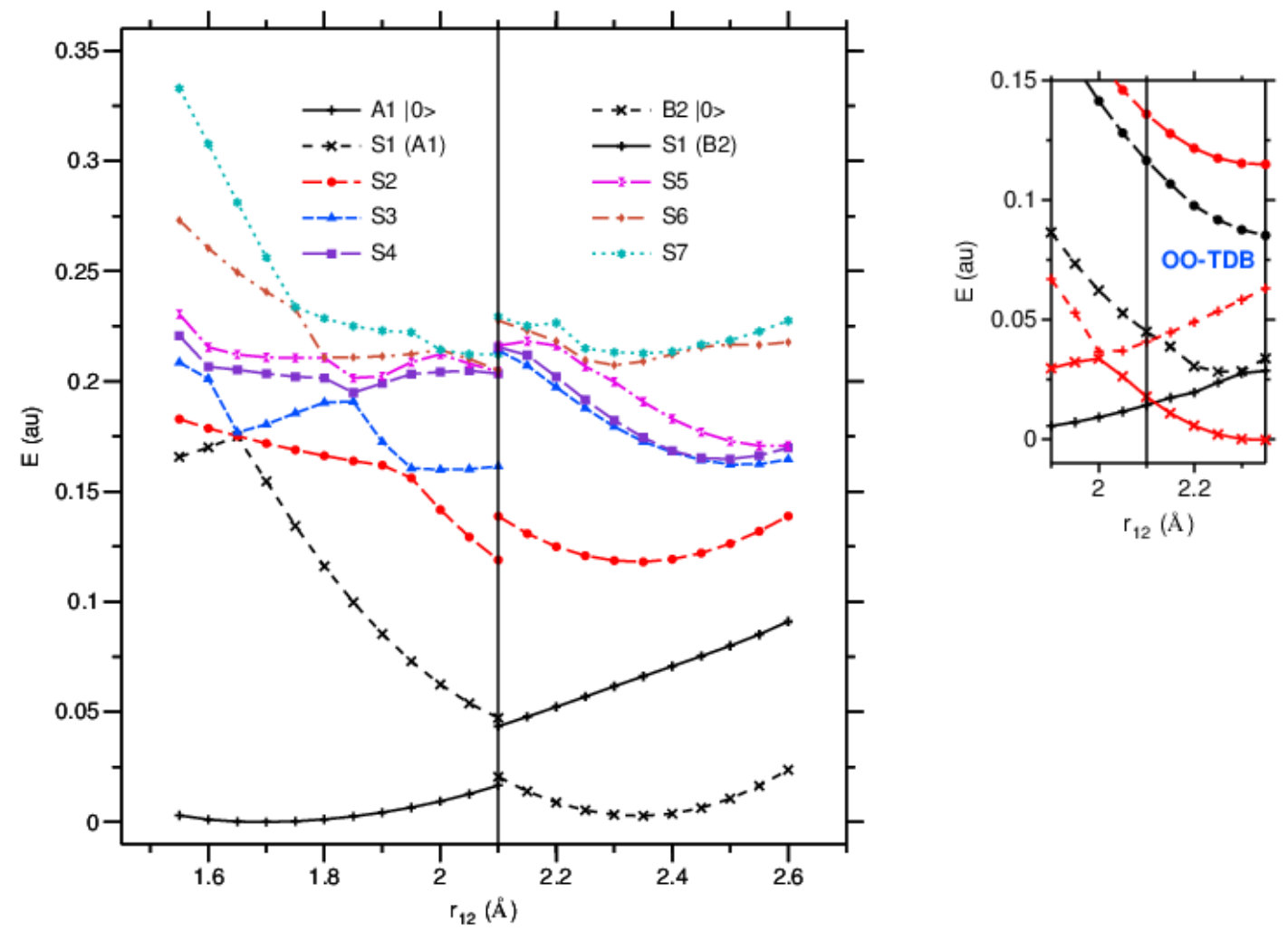

Figure 7 - Left: Energy of the lowest 9 states (Sn) of OO-TDB along the $\mathrm{r}_{12}$ coordinate. The ground state and S2 interchange for the two symmetries. Right: details of the crossing of the $A_{1}$ and $B_{2}$ eigenstates along $r_{12}$, black along the $A_{1}$ and red along the $B_{2}$ section of the potential energy surface.

Ground state minima are at $\mathrm{r}_{12} 1.692$ and $2.343 \AA$, respectively for the $\mathrm{A}_{1}$ and $\mathrm{B}_{2}$ symmetry (see Table 1). At the $\mathrm{B}_{2}$ minimum, the gap with the $\mathrm{A}_{1}$ state is $1.72 \mathrm{eV}\left(13906 \mathrm{~cm}^{-1}\right)$ and that with the quartet is $3.14 \mathrm{eV}$ (25305 $\left.\mathrm{cm}^{-1}\right)$. At the $A_{1}$ minimum the gap with the $B_{2}$ state is $4.21 \mathrm{eV}\left(33942 \mathrm{~cm}^{-1}\right)$ and that with the quartet $6.97 \mathrm{eV}$ $\left(56236 \mathrm{~cm}^{-1}\right)$. In a wide series of EOM-SF calculations reported in ref. [80], at the $\mathrm{B}_{2}$ minimum the gap with the $A_{1}$ doublet was found in the range 1.38-1.60 eV and at the $A_{1}$ minimum the gap with the $B_{2}$ in the range 4.70-4.90 eV, in reasonable agreement with our data.

The value of $r_{12}$ at the minimum in the $B_{2}$ state is about the same than for the MM-TDB species; on the contrary, in the $A_{1}$ state we have a much smaller value. Indeed, the $A_{1}$ geometry is characterized by a small value of $r_{12}$ and by strong interactions between the unpaired electrons, which are found much closer to each other with respect to MM-TDB. Consequently, the quartet is found well above the doublets and it is the eighth state (DDCI 7) at the $\mathrm{A}_{1}$ absolute minimum. For the same reason, also the energy gaps between the two doublet states and between the doublets and the quartet are always larger than for the MM-DTB system.

In the right panel of Figure 7 we focus on the region where the $A_{1}$ and $B_{2}$ doublets cross each other. In fact, while the vertical line identifies the threshold where the two states invert their relative energy position, 
crossing between them along $\mathrm{r}_{12}$ occurs at different values of the remaining internal coordinates. This figure reports the energies obtained by geometry optimization for the $A_{1}$ and $B_{2}$ states even where they are no more the ground state. Thus, considering the $\mathrm{B}_{2}$ state (red lines) the crossing with the upper $\mathrm{A}_{1}$ state occurs at $r_{12} \cong 2.0 \AA$, while for the $A_{1}$ state (black lines) the crossing with $B_{2}$ occurs at $r_{12} \cong 2.3 \AA$.

\section{Conclusions}

The present paper reports and analyzes the main aspects of a comprehensive computational study of three prototypical tri-radicals, TMB, MM-TDB and OO-TDB, which differ both in the nature $(\pi$ or $\sigma)$ and in the relative positions of the magnetic orbitals. More precisely, TMB is a $\pi \pi \pi$ tri-radical with equally spaced magnetic sites, MM-TDB still bears equally spaced magnetic sites but is a $\sigma \sigma \sigma$ tri-radical, whereas in OOTDB, again a $\sigma \sigma \sigma$ tri-radical, the three unpaired electrons are on neighboring atoms.

In spite of the close geometric similarity, the ground electronic states of MM-TDB and TMB are a doublet and a quartet, respectively: unpaired electrons are, in fact, much closer in the $\sigma \sigma \sigma$ than in the $\pi \pi \pi$ species and this stabilizes the doublet states with respect to the quartet state.

A simple Hubbard model Hamiltonian including an effective exchange confirms the above picture and permits a rationalization of the relative stability of different electronic states in terms of well-defined electronic interactions. The effective parameters of the model Hamiltonian are related to the geometrical differences between the various species, also for the two lowest doublet states, which undergo Jahn-Teller distortions that, for TMB and MM-TDB, breaks the $\mathrm{D}_{3 \mathrm{~h}}$ symmetry to $\mathrm{C}_{2 \mathrm{v}}$ and removes the degeneration.

However, the model Hamiltonian is unable to give accurate values of the energy separation between the different spin states. To this end we have employed the well-tested DDCI approach. For TMB we have thus investigated on the contribution of various classes of excitations added to the reference minimal CASCI $(3,3)$ calculation. For MM-TDB and OO-TDB, DDCI calculations have been applied to the study of the lowest states as a function of the $\mathrm{C}-\mathrm{C}$ distance, whose value determines the $\mathrm{A}_{1}$ or $\mathrm{B}_{2}$ character of the doublet ground state. This has evidenced the differences between MM-TDB and OO-TDB, with the largest energy separation for the second and the very large quartet doublet gap when the $\mathrm{A}_{1}$ state is the ground state.

Together with the intrinsic interest of the studied systems, our work paves the route, in our opinion, toward the quantitative evaluation of the relative stability of different magnetic states in poly-radicals of current technological interest and to the interpretation of general trends in terms of well-defined electronic interactions.

\section{Supplementary Material}

See Supplementary Material for the Hubbard and the minimal basis CI matrices, for the CSPA convergence, for the contribution of the different classes of excitation for TMB $\mathrm{C}_{2 \mathrm{v}}$ and for the figure of the full DDCI enrgy of the lowest nine states of MM-TDB and OO-TDB. 


\section{References}

[1] O. Kahn, Molecular Magnetism (VCH, New York, 1993).

[2] A. Rajca, Chem. Rev. 94, 871 (1994).

[3] J. M. D. Coey, J. Magn. Magn. Mater. 226, 2107 (2001).

[4] E. Coronado, P. Delhaès, D. Gatteschi, and J. S. Milles, Molecular Magnetism: From Molecular Assemblies to the Devices (Kluwer Academic Publishers, Dordrecht, 1996).

[5] D. Gatteschi, R. Sessoli, and J. Villain, Molecular nanomagnets (Oxford University Press, New York, 2006).

[6] S. Goswami, A. K. Mondal, and S. Konar, Inorg. Chem. Front. 2, 687 (2015).

[7] S. T. Liddle, and J. van Slageren, Chem. Soc. Rev. 44, 6655 (2015).

[8] Z. Zeng, X. Shi, C. Chi, J. T. Lopez Navarrete, J. Casado, and J. Wu, Chem. Soc. Rev. 44, 6578 (2015).

[9] S. N. Datta, C. O. Trindle, and F. Illas, Theoretical and Computational Aspects of Magnetic Organic Molecules (World Scientific Publishing Co. Pte. Ltd., Singapore, 2014).

[10] S. Sanvito, Chem. Soc. Rev. 40, 3336 (2011).

[11] J. M. Clemente-Juan, E. Coronado, and A. Gaita-Arino, Chem. Soc. Rev. 41, 7464 (2012).

[12] H. Gu, X. Zhang, H. Wei, Y. Huang, S. Wei, and Z. Guo, Chem. Soc. Rev. 42, 5907 (2013).

[13] H. Atsufumi, and T. Koki, J. Phys. D: Applied Physics 47, 193001 (2014).

[14] E. V. Gomonay, and V. M. Loktev, Low Temperature Physics 40, 17 (2014).

[15] A. Kazuya, Semicond. Sci. Tech. 29, 043002 (2014).

[16] S. Y. Quek, and K. H. Khoo, Acc. Chem. Res. 47, 3250 (2014).

[17] R. Naaman, and D. H. Waldeck, Annu. Rev. Phys. Chem. 66, 263 (2015).

[18] A. Yasuo, Jpn. J. Appl. Phys. 54, 070101 (2015).

[19] S. Shil, D. Bhattacharya, A. Misra, and D. J. Klein, Phys. Chem. Chem. Phys. 17, 23378 (2015).

[20] I. d. P. R. Moreira, C. de Graaf, and V. Barone, Theor. Chem. Acc. 104, 265 (2000).

[21] A. I. Krylov, J. Phys. Chem. A 109, 10638 (2005).

[22] B. D. Koivisto, and R. G. Hicks, Coord. Chem. Rev. 249, 2612 (2005).

[23] T. Itoh, K. Hirai, and H. Tomioka, B. Chem. Soc. Jpn. 80, 138 (2007).

[24] J. Lee, E. Lee, S. Kim, G. S. Bang, D. A. Shultz, R. D. Schmidt, M. D. E. Forbes, and H. Lee, Angew. Chem. Int. Ed. 50, 4414 (2011).

[25] W. C. Lineberger, and T. W. Weston, Phys. Chem. Chem. Phys. 13, 11792 (2011).

[26] S. V. Chapyshev, Russ. Chem. B. 60, 1274 (2012).

[27] M. Abe, Chem. Rev. 113, 7011 (2013).

[28] M. Winkler, and W. Sander, Acc. Chem. Res. 47, 31 (2014).

[29] J. P. Malrieu, R. Caballol, C. J. Calzado, C. de Graaf, and N. Guihéry, Chem. Rev. 114, 429 (2014).

[30] G. Gryn'ova, M. L. Coote, and C. Corminboeuf, Wiley Interdisciplinary Reviews: Comput. Molec. Science 5, 440 (2015).

[31] N. M. Gallagher, A. Olankitwanit, and A. Rajca, J. Org. Chem. 80, 1291 (2015).

[32] Y. Tsuji, R. Hoffmann, M. Strange, and G. C. Solomon, P. Natl. Acad. Sci. USA 113, E413 (2016).

[33] A. K. Pal, S. Hansda, and S. N. Datta, J. Phys. Chem. A 119, 2176 (2015).

[34] C. Felser, G. H. Fecher, and B. Balke, Angew. Chem. Int. Ed. 46, 668 (2007).

[35] T. Jahnert, M. D. Hager, and U. S. Schubert, J. Mater. Chem. A 2, 15234 (2014).

[36] P. Neuhaus, and W. Sander, Angew. Chem. Int. Ed. 49, 7277 (2010).

[37] A. Rajca, A. Olankitwanit, Y. Wang, P. J. Boratyński, M. Pink, and S. Rajca, J. Am. Chem. Soc. 135, 18205 (2013). 
[38] E. Coulaud, D. Hagebaum-Reignier, D. Siri, P. Tordo, and N. Ferre, Phys. Chem. Chem. Phys. 14, 5504 (2012).

[39] J. P. Malrieu, and G. Trinquier, J. Phys. Chem. A 116, 8226 (2012).

[40] M. E. Ali, V. Staemmler, F. Illas, and P. M. Oppeneer, J. Chem. Theory Comput. 9, 5216 (2013).

[41] Y. Liu, F. A. Villamena, A. Rockenbauer, Y. Song, and J. L. Zweier, J. Am. Chem. Soc. 135, 2350 (2013).

[42] A. K. Pal, D. R. Mañeru, I. A. Latif, I. d. P. R. Moreira, F. Illas, and S. N. Datta, Theor. Chem. Acc. 133, 1472 (2014).

[43] A. Olankitwanit, M. Pink, S. Rajca, and A. Rajca, J. Am. Chem. Soc. 136, 14277 (2014).

[44] X. Zhu, and Y. Aoki, J. Comput. Chem. 36, 1232 (2015).

[45] V. Barone, C. Boilleau, I. Cacelli, A. Ferretti, S. Monti, and G. Prampolini, J. Chem. Theory Comput. 9, 300 (2013).

[46] V. Barone, C. Boilleau, I. Cacelli, A. Ferretti, and G. Prampolini, J. Chem. Theory Comput. 9, 1958 (2013).

[47] I. Cacelli, A. Ferretti, G. Prampolini, and V. Barone, J. Chem. Theory Comput. 11, 2024 (2015).

[48] A. I. Krylov, Rev. Comput. Chem. in press (2016).

[49] C. J. Cramer, and D. G. Truhlar, Phys. Chem. Chem. Phys. 11, 10757 (2009).

[50] A. Bencini, and F. Totti, J. Chem. Theory Comput. 5, 144 (2009).

[51] F. Neese, Coord. Chem. Rev. 253, 526 (2009).

[52] A. Caneschi, D. Gatteschi, and F. Totti, Coord. Chem. Rev. 289-290, 357 (2015).

[53] Y. Shao, and M. Head-Gordon, J. Chem. Phys. 118, 4803 (2003).

[54] F. Wang, and T. Ziegler, J. Chem. Phys. 121, 12191 (2004).

[55] Z. Rinkevicius, O. Vahtras, and H. Ågren, J. Chem. Phys. 133 (2010).

[56] Y. A. Bernard, Y. Shao, and A. I. Krylov, J. Chem. Phys. 136, 204103 (2012).

[57] C. Angeli, and C. J. Calzado, J. Chem. Phys. 137, 034104 (2012).

[58] C. J. Calzado, C. Angeli, C. de Graaf, and R. Caballol, Theor. Chem. Acc. 128, 505 (2011).

[59] S. Chattopadhyay, U. S. Mahapatra, and R. K. Chaudhuri, Chem. Phys. 401, 15 (2012).

[60] A. I. Krylov, Chem. Phys. Lett. 338, 375 (2001).

[61] D. Casanova, and M. Head-Gordon, Phys. Chem. Chem. Phys. 14, 9779 (2009).

[62] N. J. Mayhall, and M. Head-Gordon, J. Chem. Theory Comput. 10, 589 (2014).

[63] N. J. Mayhall, and M. Head-Gordon, J. Phys. Chem. Lett. 6, 1982 (2015).

[64] A. I. Krylov, Acc. Chem. Res. 39, 83 (2006).

[65] J. Miralles, O. Castell, R. Caballol, and J.-P. Malrieu, Chem. Phys. 172, 33 (1993).

[66] J. Miralles, J. P. Daudey, and R. Caballol, Chem. Phys. Lett. 198, 555 (1992).

[67] N. Suaud, R. Ruamps, N. Guihéry, and J.-P. Malrieu, Journal of Chemical Theory and Computation 8, 4127 (2012).

[68] V. Barone, I. Cacelli, A. Ferretti, and M. Girlanda, J. Chem. Phys. 128, 174303 (2008).

[69] V. Barone, I. Cacelli, A. Ferretti, and G. Prampolini, Phys. Chem. Chem. Phys. 11, 3854 (2009).

[70] V. Barone, I. Cacelli, A. Ferretti, S. Monti, and G. Prampolini, J. Chem. Theory Comput. 7, 699 (2011).

[71] V. Barone, I. Cacelli, A. Ferretti, S. Monti, and G. Prampolini, Phys. Chem. Chem. Phys. 13, 4709 (2011).

[72] J. Veciana, C. Rovira, N. Ventosa, M. I. Crespo, and F. Palacio, J. Am. Chem. Soc. 115, 57 (1993).

[73] C. R. Kemnitz, R. R. Squires, and W. T. Borden, J. Am. Chem. Soc. 119, 6564 (1997).

[74] H. M. T. Nguyen, A. Dutta, K. Morokuma, and M. T. Nguyen, J. Chem. Phys. 122, 154308 (2005).

[75] T. Höltzl, T. Veszprémi, and M. T. Nguyen, Chem. Phys. Lett. 499, 26 (2010). 
[76] H. A. Lardin, J. J. Nash, and P. G. Wenthold, J. Am. Chem. Soc. 124, 12612 (2002).

[77] L. V. Slipchenko, and A. I. Krylov, J. Chem. Phys. 118, 9614 (2003).

[78] S. Venkataramani, M. Winkler, and W. Sander, Angew. Chem. Int. Ed. 44, 6306 (2005).

[79] A.-M. C. Cristian, Y. Shao, and A. I. Krylov, J. Phys. Chem. A 108, 6581 (2004).

[80] L. Koziol, M. Winkler, N. K., S. Venkataramani, W. Sander, and A. I. Krylov, J. Phys. Chem. A 111, 5071 (2007).

[81] S. Chattopadhyay, R. K. Chaudhuri, and K. F. Freed, J. Phys. Chem. A 115, 3665 (2011).

[82] M. J. Frisch, and et al, (Gaussian Inc., Pittsburgh, PA, 2009).

[83] M. W. Schmidt, K. K. Baldridge, J. A. Boats, S. T. Elbert, M. S. Gordon, J. H. Jensen, S. Koseki, N. Matsunaga, K. A. Nguyen, S. J. Su, T. L. Windus, M. Dupuis, and J. A. Montgomery, J. Comput. Chem. 14, 1347 (1993).

[84] C. J. Calzado, J. Cabrero, J.-P. Malrieu, and R. Caballol, J. Chem. Phys. 116, 2728 (2002). 